\title{
EULAR 2012 in Berlin - auf einen Blick
}

\author{
Die Highlights des diesjährigen Kongresses
}

Vom 6. bis 9. Juni 2012 fand der diesjährige EULAR Kongress in Berlin statt. Mit ca. 15.000 Teilnehmern - sehr viele aus dem Osten Europas - aber etwas weniger Satelliten-Symposien als zuletzt. Eine Zurückhaltung, die als Zeichen der angespannten wirtschaftlichen Situation zu sehen ist.

Der Präsident, Prof. Maxime Dougados begrüßte bei der Eröffnung - an der neben Wissenschaftlern und praktizierende Rheumatologen auch Selbsthilfegruppen und Health Professionals teilnahmen - diesmal relativ viele niedergelassene Ärzte als Ausdruck der Bedeutung rheumatischer Erkrankungen.

Rund 350 eingeladene Sprecher präsentierten in diesen vier Tagen eine Flut von neuen Daten und Erkenntnissen. Wesentlicher Schwerpunkt wurde auf die tägliche Poster-Tour gelegt, bei der ausgesuchte Poster diskutiert wurden. Der Andrang an diesen Veranstaltungen war sehr groß, so z. B. bei Themen wie Verbesserung der Lebensqualität und Optionen zur Therapieanpassung bei Biologika.

\section{Highlights}

Nur einige Highlights streiflichartig herausgegriffen: TNF-Alpha-Blocker führen zu einer signifikanten Reduktion von kardiovaskulären Ereignissen bei rheumatoiden Arthritis-Patienten - verglichen mit Methotrexat oder anderen DMARDs. Dies wurde in einer retrospektiven Studie an 100.000 Patienten dokumentiert. Man kann daraus postulieren, dass die effektive Unterdrückung entzündlicher Manifestationen einen wesentlichen Beitrag der anti-TNF-Therapie darstellt und hier für die Angiologie noch ein weites Indikationsfeld geöffnet werden kann.

Auch dem Begriff der „Near Remission" wurde ein Symposium gewidmet, welches aufzeigte, dass die „Near Remission" häufiger gefunden werden kann als die ACR/ EULAR Remission-Kriterien in der Früharthritis. „Near Remission“ umfasst auch radiologische Progression über drei Jahre in einer Früharthritis und berücksichtigt besonders die berufliche $\mathrm{Si}$ tuation des Patienten.

Spannend auch ein Beitrag über systemische Steroide in hoher Dosierung, wobei früher mehr auf den Erfolg als auf die Nebenwirkungen hingewiesen wurde. Die Nebenwirkungen müssten mehr in das Bewusstsein gerückt werden!

\section{Umfrage}

Interessant eine Befragung an 256 britischen Medizinstudenten im Rahmen einer allgemeinen Umfrage. Von den Studenten zeigten nur neun Prozent Interesse an der Rheumatologie in den letzten zwei Jahren des Studiums oder zum Beginn der Ausbildung im Krankenhaus. Als TopInteressensgebiet wurden genannt: Akutmedizin, Notfallmedizin und Chirurgie. Erstaunlich ist dabei, dass 60 Prozent der Studenten berichteten, dass sie nur maximal drei Wochen Rheumatologie in den letzten drei Jahren ihre Ausbildung präsentiert bekommen haben. Die Forderung: Der Rheumatologie muss mehr Bedeutung beigemessen werden!

\section{Head to Head-Studien}

Sehr interessant die „Head to Head“ Studien - so die ADACTA (Adalimumab, Actemra), die Tocilizumab Monotherapie und Adalimumab verglichen haben, wobei Tocilizumab ein besseres Outcome hatte. Ebenso die AMPLE-Studie, Abatacept versus Adalimumab bei biologisch naiv RAPatienten mit Methotrexat Backround. Hier zeigte Abatacept ein etwas besseres Outcome, wobei die Nebenwirkungsrate aber auch etwas höher lag. Die Forderung der Autoren daraus: Wir brauchen unbedingt „Head to Head“ Studien, um die optimale Betreuung unserer Patienten verantworten zu können (Dr. Michael Schiff, Dr. Cem Gabay).

In der Abstract-Session über Osteoarthritis gab es auch einige Highlights: einerseits die Bedeutung der Röntgendokumentation in der Progression und andererseits der Hinweis in der Outcome Science Session, dass Adalimumab die Knochendestruktion bei Fingerpolyarthrosen minimiert. Besonders bei erosiven Polyarthrosen ist diese Option weiter zu verfolgen (Dr. Ruth Wittoek).

Interessant die PSUMMET 1-Studie, präsentiert von Prof. Jan McInnes, durchgeführt mit 45 oder $90 \mathrm{mg}$ Ustekinumab bei der Psoriasis-Arthritis. Sie zeigte ein sehr gutes Ansprechen sowohl auf entzündliche Veränderungen im Rahmen der Arthritis und bei der Enthesitis, als auch auf die Hautveränderungen. Besonders das Ansprechen einer Enthesitis und die Besserung der Hautveränderungen verlangen, ein größeres Augenmerk auf dieses Biologikum zu lenken.

\section{Interessante Entwicklungen}

Sehr bedeutende Fortschritte sind noch in der Pipeline bei den sogenannten kleinen Zytokinen, insbesondere der JAK-Pathways (Janus Kinase-JAK- Stimulation und Produktion) - Produktion pro inflammatorischer Proteine sowie Zytokine und Chemokine und damit eine Perpetuierung der Gelenksentzündungen und Destruktionen. Diese Entwicklung sollte man interessiert verfolgen!

Sehr aktuell auch die Diskussion in der Primary Care Session (work and workability in rheumatic diseases), wo insbesondere auf die Morgensteifigkeit großer Wert gelegt wurde. Von den 534 befragten Patienten waren 59 Prozent noch voll im Arbeitsprozess, 24 Prozent in Teilarbeit; insgesamt waren neun Prozent Selbständige. Sie alle bezeichneten die Morgensteifigkeit als wesentlichen Indikator der Minderung der Lebensqualität - in vielen Ländern auch häufigste Ursache einer Frühinvalidisierung. Die Forderung daher, dass die Morgensteifigkeit als ein wesentliches Kriterium in den ACR und EULAR-Kriterien einbezogen werden sollten!

Auch der Gicht und allen ihren Frühformen einschließlich Übergewicht, Hypertonie, Nierenschädigung und frühzeitige Herzkranzgefäß-Beteiligung wurde breiter Raum gewidmet.

Hervorzuheben wäre noch das neu geschaffene „WIN Track“ (WIN steht für „What Is New"), wobei in 18 Lectures interessante Highlights und Entwicklungen von namhaften Vortragenden präsentiert wurden.

Zusammenfassend hat die Rheumatologie nichts an ihrer Dynamik eingebüßt, im Gegenteil immer neue Details und Aspekte werden in den Vordergrund gestellt. 